\title{
THE RESIDUAL FINITENESS OF $H N N$-EXTENSIONS AND GENERALIZED FREE PRODUCTS OF NILPOTENT GROUPS: A CHARACTERIZATION
}

\author{
E. RAPTIS and D. VARSOS \\ (Received 30 March 1990; revised 6 June 1991)
}

Communicated by $\mathrm{H}$. Lausch

\begin{abstract}
We study the residual finiteness of free products with amalgamations and $H N N$-extensions of finitely generated nilpotent groups. We give a characterization in terms of certain conditions satisfied by the associated subgroups. In particular the residual finiteness of these groups implies the possibility of extending the isomorphism of the associated subgroups to an isomorphism of their isolated closures in suitable overgroups of the factors (or the base group in case of $H N N$ extensions).
\end{abstract}

1991 Mathematics subject classification (Amer. Math. Soc.): 20 E 06, 20 E 26.

\section{Introduction}

A group $G$ is called residually finite $(\mathscr{R F})$ if the intersection of all normal subgroups of finite index in $G$ is trivial. If one considers generalized free products $G=\langle K * L \mid A=B, \varphi\rangle \quad(H N N$-extensions $G=\langle t, K \mid A t=B, \varphi\rangle)$ the problem is to find conditions on the constituents of these constructions, that is, $K, L, A, B, \varphi(K, A, B, \varphi)$ such that the group $G$ is residually finite. G. Baumslag in [3] gives a sufficient condition for the amalgamated free product $G=\langle K * L \mid A=B, \varphi\rangle$ to be residually finite (Proposition 2 of [3]) and applies it to the case where $K$ and $L$ are finitely generated torsion free nilpotent groups.

In [1], in the case where, in the $H N N$-extension $G=\left\langle t, K \mid t^{-1} A t=B, \varphi\right\rangle$,

(C) 1992 Australian Mathematical Society 0263-6115/92 \$A2.00+0.00 
the base group $K$ is a finitely generated abelian group, a simple necessary and sufficient condition for the group $G$ to be residually finite was given. In the present paper we generalize the results of [1] to the case where the base group is a finitely generated nilpotent group.

M. Shirvani, in [8] and [9], gives a necessary condition for an amalgamated free product or an $H N N$-extension to be residually finite in the case where the factors of the amalgamated free product or the base group of the $H N N$-extension satisfy a non-trivial law. Here we give an independent proof of these results of Shirvani in the special case, where the factors of the amalgamated free product or the base group of the $H N N$-extension are finitely generated nilpotent groups.

The concept of filtration (Definition 4 below) was introduced by G. Baumslag in [3] and used to prove that the existence of a suitable filtration implies the residual finiteness of the amalgamated free product of residually finite groups. Here we prove that the existence of suitable filtrations depends upon internal conditions satisfied by the groups (factors, base group, associated subgroups and $\varphi$ ) which take part in the relative constructions.

Our main results are contained in the following Theorems:

THeOREM $4^{*}$. Let $K, L$ be finitely generated nilpotent groups, $A \leq K$, $B \leq L$ and $\varphi: A \rightarrow B$ an isomorphism. The free product $G=\langle K * L| A=$ $B, \varphi\rangle$ of $K$ and $L$ with amalgamated subgroups $A$ and $B$ is residually finite if and only if there exist torsion-free normal subgroups $N \triangleleft K$ and $M \triangleleft L$ of finite index in $K$ and $L$ respectively such that $(A \cap N) \varphi=B \cap M$ and $A \cap N, B \cap M$ are isolated in $N$ and $M$ respectively.

THEOREM $5^{*}$. Let $K$ be a finitely generated nilpotent group, $A, B$ proper subgroups of $K$ and $\varphi: A \rightarrow B$ an isomorphism. The $H N N$-extension $G=$ $\left\langle t, K \mid t^{-1} A t=B, \varphi\right\rangle$ of $K$ with associated subgroups $A$ and $B$ is residually finite if and only if there exists a torsion free normal subgroup $N \triangleleft K$ of finite index in such $K$ that $(A \cap N) \varphi=B \cap N$ and $A \cap N, B \cap N$ are isolated in $N$.

THEOREM 6. Let $K$ and $L$ be finitely generated torsion-free nilpotent groups, $A \leq K, B \leq L$ and $\varphi: A \rightarrow B$ an isomorphism. Suppose that there exists an $(A, B, \varphi)$-filtration $\left(N_{i}, M_{i}\right)_{i \in I}$ of the pair $(K, L)$ such that the subgroups $N_{i}, M_{i}, i \in I$, are of finite index in $K$ and $L$ respectively. Then there exist finitely generated torsion-free nilpotent groups $X$ and $Y$ such that $K$ and $L$ are subgroups of finite index in $X$ and $Y$ respectively, and an isomorphism $\theta: i_{X}(A) \rightarrow i_{Y}(B)$ such that $\theta_{\mid A}=\varphi$, where $i_{X}(A)$ and $i_{Y}(B)$ are the isolated closures of $A$ and $B$ in $X$ and $Y$ respectively. 
Theorems $4^{*}$ and $5^{*}$ are in some way parallel. So their proofs are similar and we stress their common features and their technical differences.

\section{Definitions and some known facts}

In this section we give some definitions and elementary facts. For more details we refer to [1] and [2].

Definitions. 1. Let $G$ be a group and $H \leq G$. The subgroup $H$ is called isolated in $G$ if whenever $g^{n} \in H$ for $g \in G$ and $n \in \mathbb{N}$, we have $g \in H$. It is easy to prove that the intersection of a family of isolated subgroup in $G$ is also an isolated subgroup in $G$.

2. The intersection of all isolated subgroups in a group $G$ which contain the subgroup $H$ is called the isolated closure of the subgroup $H$ in $G$, and it is denoted by $i_{G}(H)$. Elementary facts and properties of isolated subgroups are contained essentially in [7, Vol. II, §66] or in [2].

3. Let $K, L$ be two groups, $A \leq K, B \leq L, \varphi: A \rightarrow B$ is isomorphism and $N \triangleleft K, M \triangleleft L$. The pair $(N, M)$ is called $\varphi$-compatible, if $(A \cap N) \varphi=B \cap M$. Let $\left(N_{i}, M_{i}\right)_{i \in I}$ be a family of $\varphi$-compatible pairs. It is easy to see that the pair $\left(\bigcap_{i \in I} N_{i}, \bigcap_{i \in I} M_{i}\right)$ is $\varphi$-compatible.

4. The family $\left(N_{i}, M_{i}\right)_{i \in I}$ of $\varphi$-compatible pairs is called an $(A, B, \varphi)$ filtration of the pair $(K, L)$ if $\bigcap_{i \in I} N_{i}=1, \bigcap_{i \in I} M_{i}=1$ and $\bigcap_{i \in I} N_{i} A=A$, $\bigcap_{i \in I} M_{i} B=B$. In the case where $K=L$ and $N_{i}=M_{i}, i \in I$, the family $\left(N_{i}\right)_{i \in I}$ is called an $(A, B, \varphi)$-filtration of $K$.

Let $(N, M)$ be a $\varphi$-compatible pair of the pair $(K, L)$. Then the isomorphism $\varphi$ induces in a natural way an isomorphism $\varphi^{*}: A / A \cap N \rightarrow B / B \cap M$. Let $\bar{\varphi}$ be the composition of the natural isomorphisms

$$
A N / N \rightarrow A / A \cap N \rightarrow B / B \cap M \rightarrow B M / M .
$$

It is easy to see that $(a N) \bar{\varphi}=a \varphi M$. So if $G=\langle K * L \mid A=B, \varphi\rangle$ is the free product with amalgamation of $K$ and $L$ with amalgamated subgroups $A$ and $B$, and $(N, M)$ is a $\varphi$-compatible pair; then we can define the free product with amalgamation

$$
\bar{G}=\langle K / N * L / M \mid A N / N=B M / M, \bar{\varphi}\rangle
$$

and the natural epimorphism $\theta: G \rightarrow \bar{G}$ which extends the natural epimorphisms $K \rightarrow K / N$ and $L \rightarrow L / M$. Clearly $\operatorname{Ker} \theta=\langle N, M\rangle^{G}$, the normal closure of $\langle N, M\rangle$ in $G$.

Similarly, if $G=\left\langle t, K \mid t^{-1} A t=B, \varphi\right\rangle$ is the $H N N$-extension of the group $K$ with associated subgroups $A$ and $B$ and $N$ is a $\varphi$-compatible subgroup of $K$, then we can define the $H N N$-extension

$$
\bar{G}=\left\langle t_{N}, K / N \mid t^{-1} A N / N t_{N}=B N / N, \bar{\varphi}\right\rangle
$$


and the epimorphism $\theta: G \rightarrow \bar{G}$, where $t \theta=t_{N}$ and $k \theta=k N$ for $k \in K$. It is easy to see that $\operatorname{Ker} \theta=N^{G}$.

\section{Necessary and sufficient conditions for the residual finiteness of certain groups}

Lemma 1 . Let $G=\langle K * L \mid A=B, \varphi\rangle$ be the free product with amalgamation of $K$ and $L$ with amalgamated subgroups $A$ and $B,\left(N_{i}, M_{i}\right)_{i \in I}$ an $(A, B, \varphi)$-filtration of the pair $(K, L)$ and for each $i \in I$,

$$
G_{i}=\left\langle K / N_{i} * L / M_{i} \mid A N_{i} / N_{i}=B M_{i} / M_{i}, \varphi_{i}\right\rangle
$$

the corresponding induced free product with amalgamation. If each $G_{i}$ is $\mathscr{R F}$, then $G$ is $\mathscr{R F}$.

Proof. See the proof of the Proposition 2 of [3].

Lemma $1^{\prime}$. Let $G=\left\langle t, K \mid t^{-1} A t=B, \varphi\right\rangle$ be the $H N N$-extension of $K$ with associated subgroups $A$ and $B,\left(N_{i}\right)_{i \in I}$ an $(A, B, \varphi)$-filtration of $K$ and for each $i \in I$,

$$
G_{i}=\left\langle t_{i}^{-1} A N_{i} / N_{i} t_{i}=B N_{i} / N_{i}, \varphi_{i}\right\rangle
$$

the corresponding $H N N$-extension. If each $G_{i}$ is $\mathscr{R} \mathscr{F}$, then $G$ is $\mathscr{R} \mathscr{F}$.

Proof. This is Lemma 1 of [1].

LemMa 2. Let $G=\langle K * L \mid A=B, \varphi\rangle$ be the free product with amalgamation of the finitely generated nilpotent groups $K$ and $L$ with amalgamated subgroups $A$ and $B$. If the group $G$ is $\mathscr{R} \mathscr{F}$, then there exists an $(A, B, \varphi)$ filtration $\left(N_{i}, M_{i}\right)_{i \in I}$ of the pair $(K, L)$ such that $N_{i} \triangleleft_{f} K, M_{i} \triangleleft_{f} L$, $i \in I$.

(The symbol $\triangleleft_{f}$ means "is normal and of finite index in".)

Proof. Suppose that the group $G$ is $\mathscr{R} \mathscr{F}$. Let $\left(G_{i}\right)_{i \in I}$ be the family of normal subgroups of finite index in $G$. Then $\bigcap_{i \in I} G_{i}=1$. Let $N_{i}=K \cap G_{i}$ and $M_{i}=L \cap G_{i}, i \in I$. Then $N_{i} \triangleleft_{f} K, M_{i} \triangleleft_{f} L$ and $\bigcap_{i \in I} N_{i}=\bigcap_{i \in I} M_{i}=$ 1. Since $a=a \varphi, a \in A$ in $G$, we have

$$
A \cap N_{i}=A \cap K \cap G_{i}=A \cap G_{i}=B \cap G_{i}=B \cap L \cap G_{i}=B \cap M_{i} .
$$

So $\left(A \cap N_{i}\right) \varphi=B \cap M_{i}, i \in I$. Also $\bigcap_{i \in I} N_{i} A=A$ and $\bigcap_{i \in I} M_{i} B=B$. In fact, if $x \in \bigcap_{i \in I} A \backslash A$ and $y \in L \backslash B \cup \gamma_{2}(L)$, then, $g=[y, x, \ldots, x] \neq 1$, 
where $r$ is the nilpotency class of $L$. But in the free products with amalgamation $G_{i}=\left\langle K / N_{i} * L / M_{i} \mid A N_{i} / N_{i}=B M_{i} / M_{i}, \varphi_{i}\right\rangle$ we have $g \theta_{i}=\left[y M_{i}, x N_{i}, \ldots, x N_{i}\right]=\left[y M_{i}, x \varphi M_{i}, \ldots, x \varphi M_{i}\right]=1 \quad$ for all $i \in I$.

So $g \in \bigcap_{i \in I} \operatorname{Ker} \theta_{i}=\bigcap_{i \in I}\left\langle N_{i}, M_{i}\right\rangle^{G} \leq \bigcap_{i \in I} G_{i}=1$, a contradiction. So the family $\left(N_{i}, M_{i}\right)_{i \in I}$ is an $(A, B, \varphi)$-filtration of the pair $(K, L)$ such that $N_{i} \triangleleft_{f} K, M_{i} \triangleleft_{f} L, i \in I$.

Lemma $2^{\prime}$. Let $G=\left\langle t, K \mid t^{-1} A t=B, \varphi\right\rangle$ be the $H N N$-extension of the finitely generated nilpotent group $K$, the associated subgroups $A$ and $B$ being proper. Suppose that the group $G$ is $\mathscr{R} F$. Then there exists an $(A, B, \varphi)$ filtration $\left(N_{i}\right)_{i \in I}$ of $K$ such that $N_{i} \triangleleft_{f} K, i \in I$.

Proof. As in the previous Lemma, since the associated subgroups are proper subgroups of $K$, we can prove that if $\left(G_{i}\right)_{i \in I}$ is the family of normal subgroups of finite index in $G$, then the family $N_{i}=K \cap G_{i}, \mid i \in I$ is an $(A, B, \varphi)$-filtration of $K$ such that $N_{i} \triangleleft_{f} K, i \in I$.

Corollary 2.1. Let $G=\left\langle t, K \mid t^{-1} A t=B, \varphi\right\rangle$ be the $H N N$-extension of the finitely generated nilpotent group $K$. If the associated subgroups $A$ and $B$ are proper subgroups of $K$ and $B<A$ (or $A<B)$, then the group $G$ is not $\mathscr{R} F$.

Proof. Let $B<A$. If $G$ were $\mathscr{R F}$, then $A=\bigcap_{i \in I} N_{i} A=\bigcap_{i \in I} N_{i} B=B$ (Lemma 3 of [8]), a contradiction.

The assumption, in the corollary above, that the subgroups $A$ and $B$ are proper subgroups of $K$, is necessary, since if $K=A$ we have:

Proposition 3. Let $K$ be a finitely generated nilpotent group without elements of finite order, $B \leq K$ and $\varphi: K \rightarrow B$ an isomorphism. If $G=\left\langle t, K \mid t^{-1} K t=B, \varphi\right\rangle$ is the corresponding $H N N$-extension, then $G$ is $\mathscr{R} F$.

Proof. Every element $g$ of $G$ is of the form $g=t^{\mu} k t^{-\nu}, k \in K$, $\mu, \nu \in \mathbb{Z}^{+}$. The homomorphism $f: G \rightarrow\langle d\rangle \quad(\langle d\rangle$ is the infinite cyclic group) which sends $t$ to $d$ and $K$ to 1 has $\operatorname{Ker} f=K^{G}$. So we can suppose that $g \in K^{G}$ and, without any loss of generality, $g \in K$. By Proposition 2.2 of [2] there exists a prime $p$ such that $K^{p^{i}} \cap B=B^{p^{i}}$ and $\bigcap_{i \in \mathrm{N}} K^{p^{i}}=1 \quad(K$ is torsion free). So for each $i$, the subgroup $K^{p^{i}}$ is $\varphi$-compatible. Therefore 
we can define the $H N N$-extension $G_{i}=\left\langle t_{i}, K_{i} \mid t_{i}^{-1} K_{i} t_{i}=B_{i}, \varphi_{i}\right\rangle$ where $K_{i}=K / K^{p^{i}}, B_{i}=B / K^{p^{i}} \cap B \cong B K^{p^{i}} / K^{p^{i}}$. Each $G_{i}$ is $\mathscr{R} \mathscr{F}$, so the group $G$ is $\mathscr{R F}$, since for $g \in K$ we can find an integer $i$ such that $g \notin K^{p^{i}}$ $\left(\bigcap_{i \in I} K^{p^{i}}=1\right)$.

Theorem 4. Let $G=\langle K * L \mid A=B, \varphi\rangle$ be the free product with amalgamation of the finitely generated nilpotent groups $K$ and $L$ with amalgamated subgroups $A$ and $B$. The group $G$ is $\mathscr{R} F$ if and only if there exists an $(A, B, \varphi)$-filtration $\left(N_{i}, M_{i}\right)_{i \in I}$ of the pair $(K, L)$ such that $N_{i} \triangleleft_{f} K$, $M_{i} \triangleleft_{f} L, i \in I$.

Proof. This is a combination of Lemmas 1 and 2, since the free product with amalgamation of finite groups is $\mathscr{R} \mathscr{F}$ (Theorem 2 of [3]).

Theorem 5. Let $G=\left\langle t, K \mid t^{-1} A t=B, \varphi\right\rangle$ be the $H N N$-extension of the finitely generated nilpotent group $K$, the associated subgroups $A$ and $B$ being proper. The group $G$ is $\mathscr{R} F$ if and only if there exists an $(A, B, \varphi)$-filtration $\left(N_{i}\right)_{i \in I}$ of $K$ such that $N_{i} \triangleleft_{f} K, i \in I$.

Proof. As above, this is immediate from Lemmas $1^{\prime}$ and $2^{\prime}$ since an $H N N$-extension of a finite group is $\mathscr{R} \mathscr{F}$ (Theorem 3.1 of [4]).

In the above two Theorems we give a necessary and sufficient condition for the residual finiteness of free products with amalgamation or $H N N$ extensions of finitely generated nilpotent groups. As we noted in the introduction this condition was proved sufficient by G. Baumslag in [3] and necessary by M. Shirvani in [8] and [9].

Using the fact that the groups we use for our contructions are finitely generated nilpotent, we can give another formulation of Theorems 4 and 5 .

THEOREM $4^{*}$. With the hypothesis of Theorem 4, the group $G$ is $\mathscr{R F}$ if and only if there exist torsion-free subgroups $N \triangleleft_{f} K$ and $M \triangleleft_{f} L$ such that $(A \cap N) \varphi=B \cap M$ and $A \cap N, B \cap M$ are isolated in $N$ and $M$ respectively.

Proof. Suppose that the group $G$ is $\mathscr{R} F$. Then there exists an $(A, B, \varphi)$ filtration $\left(N_{i}, M_{i}\right)_{i \in I}$ of $(K, L)$ such that $N_{i} \triangleleft_{f} K$ and $M_{i} \triangleleft_{f} L, i \in I$. Since $\bigcap_{i \in I} N_{i} A=A$ and $A \leq_{f} i_{K}(A)$, there exists $i_{0} \in I$ such that $N_{i_{0}} \cap$ $a A=\varnothing$ for each proper coset of $A$ in $i_{K}(A)$. Therefore $N_{i_{0}} \cap i_{K}(A) \leq A$. Similarly there exists $M_{j_{0}} \in\left(M_{i}\right)_{i \in I}$ such that $M_{j_{0}} \cap i_{L}(B) \leq B$. Let $N=$ $N_{i} \cap N_{j_{0}} \cap T$, where $T \triangleleft_{f} G$ such that $T \cap T_{K}=T \cap T_{L}=1$, and $T_{K}, T_{L}$ are 
the torsion parts of $K$ and $L$ respectively. Similarly let $M=M_{i_{0}} \cap M_{j_{0}} \cap T$. Then $(A \cap N) \varphi=B \cap M$ and $N \cap i_{K}(A) \leq A, M \cap i_{L}(B) \leq B$. But $i_{N}(A \cap N)=N \cap i_{K}(A)$. In fact, if $a \in N \cap i_{K}(A)$, then $a^{n} \in A, n \in \mathbb{N}$, so $a \in i_{N}(A \cap N)$ and $N \cap i_{K}(A) \leq i_{N}(A \cap N)$. Clearly $i_{N}(A \cap N) \leq N \cap i_{K}(A)$, and finally $i_{N}(A \cap N)=N \cap i_{K}(A)$. Similarly $i_{M}(B \cap M)=M \cap i_{L}(B)$. So $i_{N}(A \cap N)=N \cap i_{K}(A)=A \cap N, i_{M}(B \cap M)=M \cap i_{L}(B)=B \cap M$ and $A \cap N$, $B \cap M$ are isolated in $N$ and $M$ respectively.

Conversely, if there exist $N \triangleleft_{f} K, M \triangleleft_{f} L$ such that $(A \cap N) \varphi=B \cap M$ and $A \cap N, B \cap M$ are isolated in $N$ and $M$ respectively, then there exists a prime $p$ such that $\bigcap_{i \in \mathbb{N}} N^{p^{i}}=\bigcap_{i \in \mathbb{N}} M^{p^{i}}=1 \quad(N$ and $M$ are torsion-free). Furthermore

$\left(A \cap N^{p^{i}}\right) \varphi=\left(A \cap N \cap N^{p^{i}}\right) \varphi=(A \cap N)^{p^{i}} \varphi=(B \cap M)^{p^{i}}=(B \cap M) \cap M^{p^{i}}=B \cap M^{p^{i}}$.

On the other hand, since $A \cap N, B \cap M$ are isolated in $N$ and $M$, we have $i_{N}(A \cap N)=A \cap N$ and $i_{M}(B \cap M)=B \cap M$. Consequently we have $N \cap i_{K}(A)=A \cap N$ and $M \cap i_{L}(B)=B \cap M$. Moreover we have $\bigcap_{i \in \mathbb{N}} N^{p^{i}}(A \cap N)=A \cap N, \bigcap_{i \in \mathbb{N}} M^{p^{i}}(B \cap M)=B \cap M$. (N, $M$ are torsionfree and $A \cap N, B \cap M$ are isolated in $N$ and $M$ (Theorem 2.5 of [2])). Let $x \in \bigcap_{i \in \mathbb{N}} N^{p^{i}} A$. Since $N \triangleleft_{f} K$, we have $x^{\lambda} \in N, \lambda \in \mathbb{N}$, that is

$$
x^{\lambda} \in\left(\bigcap_{i \in \mathbb{N}} N^{p^{i}} A\right) \cap N=\bigcap_{i \in \mathbb{N}}\left(N^{p^{i}} A \cap N\right) \leq \bigcap_{i \in \mathbb{N}} N^{p^{i}}(A \cap N)=A \cap N .
$$

Therefore $x \in i_{K}(A)$, so $\bigcap_{i \in \mathbb{N}} N^{p^{i}} A \leq i_{K}(A)$. Consequently if $x \in \bigcap_{i \in \mathbb{N}} N^{p^{i}} A \backslash A$, we have that for each $i \in \mathbb{N}$ there exists $h_{i} \in N^{p^{i}}, a_{i} \in A$ such that $x=h_{i} a_{i}$, so $x a_{i}^{-1}=h_{i} \in N^{p^{i}}, i \in \mathbb{N}$ and $x a_{i}^{-1} \in i_{K}(A) \cap N=$ $A \cap N$, thus $x \in A$, a contradiction. Similarly $\bigcap_{i \in \mathbb{N}} M^{p^{i}} B=B$. Therefore the family $\left(N_{i}, M_{i}\right)_{i \in \mathrm{N}}$ is an $(A, B, \varphi)$-filtration of the pair $(K, L)$ and the group $G$ is $\mathscr{R} F$.

THEOREM $5^{*}$. With the hypothesis of Theorem 5 , the group $G$ is $\mathscr{R F}$ if and only if there exists a torsion-free subgroup $N \triangleleft_{f} K$ such that $(A \cap N) \varphi=$ $B \cap N$ and the subgroups $A \cap N, B \cap N$ are isolated in $N$.

Proof. The proof is similar to the proof of the previous Theorem. So we omit it.

COROLlaRy 4.1. With the hypothesis of Theorem 4, we have:

i) If the amalgamated subgroups $A$ and $B$ are of finite index in $K$ and 
$L$, then the group $G$ is $\mathscr{R F}$ if and only if there exist $N \triangleleft K, M \triangleleft L$ such that $N \triangleleft_{f} A, M \triangleleft_{f} B$ and $N \varphi=M$.

ii) If the factors $K$ and $L$ are torsion-free and the amalgamated subgroups $A$ and $B$ isolated in $K$ and $L$, then the group $G$ is $\mathscr{R} F$.

iii) If the factors $K$ and $L$ are torsion-free and there exists an isomorphism $\theta: K \rightarrow L$ such that $\theta_{\mid A}=\varphi$, then the group $G$ is $\mathscr{R} F$.

Proof. i) It is enough to note that $i_{K}(A)=K$ and $i_{L}(B)=L$, since $A$ and $B$ are of finite index in $K$ and $L$ respectively.

ii) Let $p$ be a prime greater than the nilpotency class of $K$ and $L$. If $N=K^{p}, M=L^{p}$, then from the Corollary 2.31 of [2], every element of $N$ and $M$ is of the form $k^{p}, k \in K$, and $m^{p}, m \in L$ respectively. So we have for $g \in A \cap N,(g) \varphi=\left(k^{p}\right) \varphi, k \in K$, but $k \in A$ ( $A$ is isolated), so $\left(k^{p}\right) \varphi=(k \varphi)^{p} \in B \cap M$ and finally $(A \cap N) \varphi=B \cap M$. Moreover we have $i_{N}(A \cap N)=i_{K}(A) \cap N=A \cap N$ and $i_{M}(B \cap M)=i_{L}(B) \cap M=B \cap M$. So the group $G$ is $\mathscr{R} F$.

iii) Since $\theta$ is an isomorphism of $K$ and $L$, it is easy to see that $\left(i_{K}(A)\right) \theta=i_{L}(B)$. Evidently $\left|i_{K}(A): A\right|=\left|i_{L}(B): B\right|=m$. By Corollary 2.32 of [2] there exists a positive integer $r(m, c)=r$ ( $r$ depends upon the nilpotency class $c$ of $K$ and $m$ ) such that every product of $m^{n}$ powers $(n \geq r)$ of elements of $K$ is a $m^{s}$ th power, $s=n-r$, of an element of $K$. Let $N=K^{m^{r+1}}$. Every element of $N$ is of the form $g^{m}, g \in K$. Similarly let $M=L^{m^{r+1}}$. So $N \cap i_{K}(A) \leq A, M \cap i_{L}(B) \leq B$ and $A \cap N=i_{N}(A \cap N)$, $B \cap M=i_{M}(B \cap M)$. Also

$$
(A \cap N) \varphi=(A \cap N) \theta=\left(i_{K}(A) \cap N\right) \theta=i_{L}(B) \cap M=B \cap M .
$$

Since $g \in i_{K}(A) \cap N$ implies $g=k^{m}, k \in K$, it follows that $k \in i_{K}(A)$ and $g \theta=\left(k^{m}\right) \theta=(k \theta)^{m} \in i_{L}(B) \cap M=B \cap M$.

Case ii) in the previous Corollary is Theorem 5 of [3] and case iii) is related to Theorem 10 of [3].

We have a similar Corollary for $H N N$-extensions.

COROllary 5.1. With the hypothesis of Theorem 5, we have:

i) If the associated subgroups $A$ and $B$ are of finite index in the base group $K$, then the group $G$ is $\mathscr{R F}$ if and only if there exists $H \triangleleft_{f} K$ such that $H \varphi=H$.

ii) If the base group $K$ is torsion free and the associated subgroups $A$ and $B$ isolated in $K$, then the group $G$ is $\mathscr{R F}$.

iii) If the base group $K$ is torison free and there exists an automorphism $\theta$ of $K$ such that $\theta_{\mid A}=\varphi$, then the group $G$ is $\mathscr{R F}$. 
Proof. The proof is similar to the proof of the previous Corollary, so we omit it.

Case ii) in the previous Corollary is an application of Theorem 4.2 of [4, page 189] and case iii) is derived from Lemma 4.4 of [4], since a finitely generated nilpotent group satisfies condition 4.3 of [4].

Since the case of $H N N$-extensions one of the associated subgroups can be the whole base group, Proposition 3 and Theorem $5^{*}$ give:

Corollary 5.2. The HNN-extension $G=\left\langle t, K \mid t^{-1} A t=B, \varphi\right\rangle$ of the finitely generated torsion free nilpotent group $K$ is $\mathscr{R F}$ if and only if either

i) $K=A$ (or $K=B$ ), or

ii) $A \neq K \neq B$ and there exists $N \triangleleft_{f} K$ such that $(A \cap N) \varphi=B \cap N$ and the subgroups $A \cap N$ and $B \cap N$ are isolated in $N$.

As we can see in case iii) in either of the Corollaries 4.1 and 5.1, the possibility of extending the isomorphism of the associated subgroups to an isomorphism of the factors (or to an automorphism of the base group, in the case of $H N N$-extensions) is a sufficient, condition for the residual finiteness of these groups. We shall prove that this condition is (partially) necessary in the following sense: The factors (or the base group) can be embedded in suitable finitely generated nilpotent groups such that the isomorphism of the associated subgroups comes from an isomorphism of their isolated closures in these groups.

THEOREM 6. Let $K, L$ be finitely generated torsion-free nilpotent groups with $A \leq K, B \leq L$ and $\varphi: A \rightarrow B$ an isomorphism. Suppose that there exists an $(A, B, \varphi)$-filtration $\left(N_{i}, M_{i}\right)_{i \in I}$ of $(K, L)$ such that $N_{i} \triangleleft_{f} K$, $M_{i} \triangleleft_{f} L, i \in I$. Then there exist finitely generated torsion free nilpotent groups $X$ and $Y$ such that $K, L$ are subgroups of finite index in $X$ and $Y$ respectively and an isomorphism $\theta: i_{X}(A) \rightarrow i_{Y}(B)$ such that $\theta_{\mid A}=\varphi$.

Proof. We have an $(A, B, \varphi)$-filtration $\left(N_{i}, M_{i}\right)_{i \in I}$ of $(K, L)$. So by Theorem $4^{*}$ there exist torsion-free subgroups $N \triangleleft_{f} K$ and $M \triangleleft_{f} L$ such that $(A \cap N) \varphi=B \cap M$ and $i_{N}(A \cap N)=A \cap N, i_{M}(B \cap M)=B \cap M$. Let $|K: N|=n$ and $|L: M|=m$. We define $X=\left\langle x \in m(K) \mid x^{n m} \in N\right\rangle$ and $Y=\left\langle y \in m(L) \mid y^{n m} \in M\right\rangle$, where $m(K)$ and $m(L)$ are the Mal'cev completion of $K$ and $L$ respectively. It is easy to see [2, page 57] that the groups $K$ and $L$ are of finite index in $X$ and $Y$ respectively $(K, L$ are finitely generated). Also the groups $X$ and $Y$ are finitely generated. Since $i_{X}(A) \cap N=i_{N}(A \cap N)=A \cap N$ and $i_{Y}(B) \cap M=i_{M}(B \cap M)=B \cap M$, we 
have $x^{n m} \in A \cap N$, for $x \in i_{X}(A)$, and $y^{n m} \in B \cap M$, for $y \in i_{M}(B)$. But the groups $N$ and $M$ are $\varphi$-compatible, so $\left(x^{n m}\right) \varphi \in B \cap M$. Let $y \in m(L)$ such that $y^{n m}=\left(x^{n m}\right) \varphi$. From the definition of $Y$ we have $y \in Y$, and $y^{n m}=\left(x^{n m}\right) \varphi \in B \cap M$ implies $y \in i_{Y}(B)$. Let $\theta: i_{X}(A) \rightarrow i_{Y}(B)$ with $(x) \theta=y$. The map $\theta$ is well defined, since $m(K)$ and $m(L)$ are divisible groups and for $\left(x^{n m}\right) \varphi \in B \cap M$ there exists unique $y \in m(L)$ such that $y^{n m}=\left(x^{n m}\right) \varphi$. Furthermore it is easy to see that $\theta$ is $1-1$ and onto. The map $\theta$ is homomorphism. Indeed from the relation $i_{X}(A) \cap N=A \cap N$ we have $i_{X}(A)=\left\langle x \in X \mid x^{n m} \in A \cap N\right\rangle=\left\langle x \in m(A \cap N) \mid x^{n m} \in A \cap N\right\rangle$, and from the relation $i_{Y}(B) \cap M=B \cap M$ we have $i_{Y}(B)=\left\langle y \in Y \mid y^{n m} \in B \cap M\right\rangle=$ $\left\langle y \in m(B \cap M) \mid y^{n m} \in B \cap M\right\rangle$, so $\theta$ is a homomorphism [2, p. 57]. Also $\theta_{\mid A}=\varphi$, since for $a \in A$ we have $a^{n m} \in A \cap N$, namely $\left(a^{n m}\right) \theta=\left(a^{n m}\right) \varphi$, which gives $(a \theta)^{n m}=(a \varphi)^{n m}$ and finally $a \theta=a \varphi$.

Similarly we can prove that, if we have a finitely generated torsion-free nilpotent group $K, A, B \leq K, \varphi: A \rightarrow B$ an isomorphism and an $(A, B, \varphi)$ filtration of $K$, then we can extend the isomorphism $\varphi$ to an isomorphism $\theta: i_{X}(A) \rightarrow i_{X}(B)$, where $X$ is a finitely generated torsion-free nilpotent overgroup of $K$.

It is worth remarking that the previous Theorem does not involve free products with amalgamation but deals with two properties of torsion free nilpotent groups. (The existence of an appropriate filtration implies the extension of isomorphisms.)

The study of the residual finiteness of free products with amalgamation and $H N N$-extensions of finitely generated nilpotent groups, in all cases above, makes use of the concept of filtration. In this way we try to see, if these groups are residually $\mathscr{X}$ where $\mathscr{Z}$ is the class of the free-by-finite groups, which we know are $\mathscr{R}$. We will give another sufficient condition, which may replace the condition of the existence of an appropriate filtration in a wide class of free products with amalgamation and $H N N$-extensions.

Definition. The group $G$ is called subgroup separable $(\mathscr{S P})$ if $\bigcap_{N \in \mathcal{N}} N H=H$, for every finitely generated subgroup $H$, where $\mathscr{N}=$ $\left\{N \mid N \triangleleft_{f} G\right\}$.

It is easy to see that the quotient group $G / H$ of a $\mathscr{S} \mathscr{S}$ group is $\mathscr{R} \mathscr{F}$ for every finitely generated normal subgroup $H$.

THEOREM 7. Let $G=\langle K * L \mid A=B, \varphi\rangle$ be the free product with amalgamation of the finitely generated $\mathscr{S S S}$ groups $K$ and $L$. Suppose that there exist finitely generated normal subgroups $N$ and $M$ of $K$ and $L$ respectively such that $N \triangleleft_{f} A, M \triangleleft_{f} B$ and $N \varphi=M$. Then the group $G$ is $\mathscr{R} F$. 
Proof. The subgroups $N$ and $M$ are $\varphi$-compatible, so we can define the free product with amalgamation

$$
\bar{G}=\langle K / N * L / M \mid A / N=B / M, \bar{\varphi}\rangle
$$

and the "natural" epimorphism $\theta: G \rightarrow \bar{G}$ with $\operatorname{Ker} \theta=N$. The group $\bar{G}$ is $\mathscr{R F}$ (Theorem 3 of [3]). So for $1 \neq g \in G$ we can assume that $g \in N=N \varphi=M$ in $G$. But $N$ and $M$ are finitely generated and $\mathscr{R F}$, therefore there exists a characteristic subgroup $N_{1}$ of $N$ of finite index in $N$ such that $g \notin N_{1}$. Let $M_{1}=N_{1} \varphi$. Since $g \notin N_{1}$, we can argue as before with $N_{1}, M_{1}$ in place of $N$ and $M$. So, $G$ is $\mathscr{R}(\mathscr{R} \mathscr{F})$, that is $G$ is $\mathscr{R F}$.

THEOREM $7^{\prime}$. Let $G=\left\langle t, K \mid t^{-1} A t=B, \varphi\right\rangle$ be an $H N N$-extension of a finitely generated $\mathscr{S S S}$ group $K$. Suppose that there exists a finitely generated normal subgroup $N$ of $K$ such that $N \triangleleft_{f} A, N \triangleleft_{f} B$ and $N \varphi=N$. Then the group $G$ is $\mathscr{R} F$.

Proof. As above, we can prove that the $H N N$-extension $G$ is $\mathscr{R F}$, since the $H N N$-extension of a finitely generated $\mathscr{R} F$ group with finite associated subgroups is $\mathscr{R} F$ (Theorem 3.1 of [4]).

COROLlaRY 7.1. i) With the hypothesis of Theorem 7, if the amalgamated subgroups $A$ and $B$ are finitely generated and normal in $K$ and $L$, then the free product with amalgamation $G=\langle K * L \mid A=B, \varphi\rangle$ is $\mathscr{R} \mathscr{F}$.

ii) With the hypothesis of Theorem $7^{\prime}$ if the associated subgroups $A$ and $B$ are equal $(A=B)$, finitely generated and normal in $K$, then the $H N N$ extension $G\left\langle t, K \mid t^{-1} A t=B, \varphi\right\rangle$ is $\mathscr{R F}$.

We note that Theorem 9 of [3] is a Corollary of 7.1 i), since finitely generated polycyclic groups are $\mathscr{S} \mathscr{S}$.

COROLlaRY 7.2. Let $K, L$ be finitely generated torsion-free nilpotent groups with $A \leq K, B \leq L$ and $\varphi: A \rightarrow B$ an isomorphism. If the subgroups $A$ and $B$ are normal in $K$ and $L$ respectively, then there exist finitely generated torsion-free nilpotent groups $X$ and $Y$ such that $K, L$ are subgroups of finite index in $X$ and $Y$ respectively and an isomorphism $\theta: i_{X}(A) \rightarrow i_{Y}(B)$ such that $\theta_{\mid A}=\varphi$.

PROoF. This is a consequence of Corollary 7.1, Lemma 2 and Theorem 6.

In [3, Theorem 4] G. Baumslag proves that the free product with amalgamation of two finitely generated torsion-free nilpotent groups is always free 
by residually finite. We prove that this is also the case for $H N N$-extensions of finitely generated torsion-free nilpotent groups.

Proposition 8. Let $G=\left\langle t, K \mid t^{-1} A t=B, \varphi\right\rangle$ be the $H N N$-extension of a finitely torsion-free nilpotent group $K$. Let $\left(N_{i}\right)_{i \in I}$ be the family of normal subgroups of finite index in $G$. If $N=\bigcap_{i \in I} N_{i}$, then $N$ is free and $N \leq K^{G}$. Moreover if $N$ is not trivial, then it is infinitely generated.

Proof. By Proposition 2.2 of [2] there exists a prime $p$ such that $A \cap K^{p^{n}}=A^{p^{n}}$ and $B \cap K^{p^{n}}=B^{p^{n}}$ for every $n \in \mathbb{N}$. If $1 \neq x \in N \cap K$, then there exists $n \in \mathbb{N}$ such that $x \notin K^{p^{n}}\left(\bigcap_{n \in \mathbb{N}} K^{p^{n}}=1\right.$, since $K$ is torsion-free). Therefore the $H N N$-extension

$$
G_{n}=\left\langle t_{n}, K / K^{p^{n}} \mid t_{n}^{-1} A K^{p^{n}} / K^{p^{n}} t_{n}=B K^{p^{n}} / K^{p^{n}}\right\rangle
$$

is a $\mathscr{R F}$ homomorphic image of $G$ with non-trivial image of $x$. So there exists $N_{i} \triangleleft_{f} G$ such that $x \in N_{i}$, a contradiction. Therefore $N \cap K=1$ and $N$ is free by a well known Theorem of Karrass and Solitar. Let $g \in G$ and the exponent sum of $t$ in $g$ be $r \neq 0$. Then $g \notin \operatorname{Ker}(i \circ \sigma)$, where $i: G \rightarrow G / K^{G} \cong\langle t\rangle$ and $\sigma:\langle t\rangle \rightarrow\left\langle t \mid t^{r+1}\right\rangle$. But $\operatorname{Ker}(i \circ \sigma) \triangleleft_{f} G$, so $g \notin N$. Therefore $N \leq K^{G}$. If $N$ is non-trivial and finitely generated, then by Theorem 9 of [6], we have that the index of the double cosets $(N, K)$ in $G$ is finite. But $|G:(N, K)|=|G: N K| \geq\left|G: K^{G}\right|=\infty$, a contradiction. Therefore $N$ is infinitely generated, and $G$ is free-by-residually finite, since $G / N$ is $\mathscr{R F}$.

In [5] (Theorem 3) J. L. Dyer notes that the requirement that the factors must be torison-free is necessary in the proof of Theorem 4 of [3]. We can adjust Dyer's example to the case of $H N N$-extensions.

Proposition 9. There exists a finitely nilpotent group $K$ with $A$ and $B$ isomorphic subgroups such that the $H N N$-extension $G=\langle t, K| t^{-1} A t=$ $B, \varphi\rangle$ is not free by residually finite.

Proof. We use Dyer's group $K$ : the group $K$ has generators $k_{1}, k_{2}, k_{3}$, $a, d$ and relations $\left[k_{i}, k_{j}\right]=1, a^{-1} k_{1} a=k_{2}, a^{-1} k_{2} a=k_{3}, a^{-1} k_{3} a=$ $k_{1} k_{2}^{-3} k_{3}^{3}, d^{p}=d^{p}=[d, a]=\left[d, k_{j}\right]=1, i, j=1,2,3, p$ any prime.

Let $A, B$ be the subgroups $A=\left\langle k_{1}, k_{2}^{p}, k_{3}, d\right\rangle, B=\left\langle k_{1}^{p} d, k_{2}, k_{3}^{p}, d\right\rangle$, and $\varphi$ the isomorphism $\varphi: A \rightarrow B$ given by $k_{1} \varphi=k_{1}^{p} d, k_{2}^{p} \varphi=k_{2}, k_{3} \varphi=$ $k_{3}^{p}, d \varphi=d$ and the group $G=\left\langle t, K \mid t^{-1} A t=B, \varphi\right\rangle$. We can see that 
$d \in \bigcap_{i \in I} N_{i}$, where $\left(N_{i}\right)_{i \in I}$ is the family of normal subgroups of finite index in $G$. So $\bigcap_{i \in I} N_{i}$ is not free.

\section{Acknowledgement}

The authors are indebted to $\mathrm{S}$. Andreadakis for helpful conversations during the preparation of this paper.

\section{References}

[1] S. Andreadakis, E. Raptis and D. Varsos, 'A characterization of residually finite $H N N$ extensions of finitely generated abelian groups', Arch. Math. 50 (1988), 495-501.

[2] G. Baumslag, Lectures on nilpotent groups, C.B.M.S. Regional Conference Series 2 (Amer. Math. Soc., Providence, R.I., 1971).

[3] G. Baumslag, 'On the residual finiteness of generalized free products of nilpotent groups', Trans. Amer. Math. Soc. 106 (1963), 193-209.

[4] B. Baumslag, M. Tretkoff, 'Residually finite $H N N$-extensions', Comm. Algebra 6 (1978), 179-194.

[5] J. L. Dyer, 'On the residual finiteness of generalized free products', Trans. Amer. Math. Soc. 133 (1968), 131-143.

[6] A. Karras, D. Solitar, 'Subgroups of $H N N$ groups and groups with one defining relation', Canad. J. Math. 23 (1971), 627-643.

[7] A. G. Kurosh, The theory of groups (Chelsea, New York 1960).

[8] M. Shirvani, 'On residually finite $H N N$-extensions', Arch. Math. 44 (1985), 110-115.

[9] M. Shirvani, 'A converse to a residual finiteness theorem of G. Baumslag', Proc. Amer. Math. Soc. 104 (1988), 703-706.

\section{University of Athens}

Panepistemiopolis

\section{Athens}

\section{Greece}

